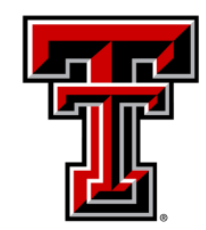

TEXAS TECH UNIVERSITY

Libraries"

\title{
ASSESSING THE ROLE OF TRAP-TO-BAND IMPACT IONIZATION AND HOLE TRANSPORT ON THE DARK CURRENTS OF 4H-SIC PHOTOCONDUCTIVE SWITCHES CONTAINING DEEP DEFECTS
}

\section{The Texas Tech community has made this publication openly available. Please share how this access benefits you. Your story matters to us.}

\begin{tabular}{|l|l|}
\hline Citation & $\begin{array}{l}\text { A. R. Chowdhury, J. C. Dickens, A. A. Neuber, and R. P. Joshi, } \\
\text { "Assessing the Role of Trap-to-Band Impact lonization and Hole } \\
\text { Transport on the Dark Currents of 4H-SiC Photoconductive Switches } \\
\text { Containing Deep Defects," Journ. Appl. Phys. 120, 245705 (2016). } \\
\text { https://doi.org/10.1063/1.4972968 }\end{array}$ \\
\hline Citable Link & $\underline{\text { https://hdl.handle.net/2346/86913 }}$ \\
\hline Terms of Use & $\begin{array}{l}\text { This article may be downloaded for personal use only. Any other } \\
\text { use requires prior permission of the author and AIP Publishing. }\end{array}$ \\
\hline
\end{tabular}




\section{Assessing the role of trap-to-band impact ionization and hole transport on the dark currents of $4 \mathrm{H}-\mathrm{SiC}$ photoconductive switches containing deep defects}

Cite as: J. Appl. Phys. 120, 245705 (2016); https://doi.org/10.1063/1.4972968

Submitted: 30 July 2016 . Accepted: 10 December 2016 . Published Online: 28 December 2016

A. R. Chowdhury, J. C. Dickens, (D. A. A. Neuber, and R. P. Joshi
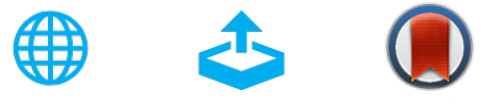

\section{ARTICLES YOU MAY BE INTERESTED IN}

Experimental characterization of impact ionization coefficients for electrons and holes in GaN grown on bulk GaN substrates

Applied Physics Letters 112, 262103 (2018); https://doi.org/10.1063/1.5031785

Analysis of high field effects on the steady-state current-voltage response of semi-insulating $4 \mathrm{H}-\mathrm{SiC}$ for photoconductive switch applications

Journal of Applied Physics 118, 095701 (2015); https://doi.org/10.1063/1.4929809

Lock-on physics in semi-insulating GaAs: Combination of trap-to-band impact ionization, moving electric fields and photon recycling

Journal of Applied Physics 123, 085703 (2018); https://doi.org/10.1063/1.5013248

Challenge us.

What are your needs for periodic signal detection?

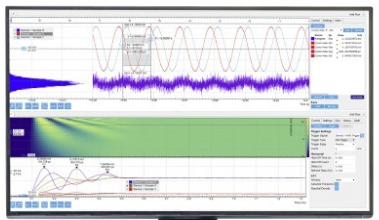

0
Zurich

- Instruments 


\title{
Assessing the role of trap-to-band impact ionization and hole transport on the dark currents of $4 \mathrm{H}-\mathrm{SiC}$ photoconductive switches containing deep defects
}

\author{
A. R. Chowdhury, J. C. Dickens, A. A. Neuber, and R. P. Joshi ${ }^{\text {a) }}$ \\ Department of Electrical and Computer Engineering, Texas Tech University, Lubbock, Texas 79409, USA
}

(Received 30 July 2016; accepted 10 December 2016; published online 28 December 2016)

\begin{abstract}
Simulation studies of the electrical response characteristics of $4 \mathrm{H}-\mathrm{SiC}$ switches containing traps are reported in the absence of photoexcitation. The focus is on trap-to-band impact ionization and the role of hole injection from the anode. Simulations show that hole-initiated ionization can be more important than the electron-initiated process. The results also underscore the role of hole injection at the high applied voltages. Our one-dimensional, time-dependent model yielded reasonable agreement with measured current-voltage data spanning over three orders of magnitude, but only when impact ionization was taken into account. Finally, the simulations predicted undulations in the device conduction current density with respect to time, due to the dynamic interplay between impact ionization, spatial electric field values, and occupancies of the trap levels. Published by AIP Publishing. [http://dx.doi.org/10.1063/1.4972968]
\end{abstract}

\section{INTRODUCTION}

Since first described by Auston in $1975,{ }^{1}$ photoconductive semiconductor switches (PCSSs) have been investigated intensively. Their unique advantages over conventional gas and mechanical switches include high speeds, fast rise times, optical isolation, compact geometry, and negligible jitter. Some relevant examples of PCSS pulsed power applications are: microwave and millimeter wave generation, impulse and ultrawideband radar, pulsed power systems, particle accelerators, directed energy systems, and trigger generator systems. $^{2,3}$

Much of the PCSS work reported to date has centered on $\mathrm{GaAs}$ or $\mathrm{Si}$, though these materials have limitations for operation at high fields, elevated temperatures, and high radiation levels. ${ }^{4} \mathrm{SiC}$ polytypes, however, appear better suited as they have greater resistance to chemicals and radiation, are stable at high temperatures, ${ }^{5}$ and have a larger saturation electron drift velocity, better thermal conductivity, and higher breakdown fields in the 3-4 MV/cm range. ${ }^{6}$ Also, for the same breakdown voltage, the on-state resistance of a $\mathrm{SiC}$ device is expected to be lower by two orders of magnitude than for $\mathrm{Si}^{7} \mathrm{SiC}$ seems to be the only technology currently available that can achieve MegaWatt output power and MegaHertz repetition rates which are of practical interest. ${ }^{8,9}$

This contribution focusses on an analysis of the processes and mechanisms expected to play a role in the operation of $4 \mathrm{H}-\mathrm{SiC}$ photoconductive switches for high pulsed power applications. ${ }^{10,11}$ These devices typically have deep levels and traps (often through deliberate irradiation) aimed at increasing the hold-off voltage and reducing the OFF-state leakage currents. The combination of traps in such semiinsulating material and operation at high applied fields can be expected to bring the following physics to the forefront:

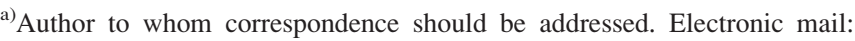
ravi.joshi@ttu.edu
}

(a) trap-to-band impact ionization initiated by electrons and/ or holes and (b) possible hole injection with the potential for instability through filamentation. Negative differential conductivity (NDC) could be associated with increasing device current as hole densities increase, while the internal polarization would lower the electric field distribution. (c) Creation of high electric fields could facilitate hole injection from the anode contact.

Though band-to-band impact ionization has been studied, the trap-to-band processes have typically been ignored. Yet in semi-insulating $\mathrm{SiC}$, the presence of traps, coupled with the lower threshold field for trap-to-band impact ionization (as compared to band-to-band ionization), would enhance this process. Hence, trap-to-band impact ionization is studied here. The non-local element of impact ionization and the role of holes are included in this analysis. The importance of non-local effects in impact ionization has been long recognized, particularly in connection with breakdown in gases, where Crooke's dark space and glow striations provide classic examples. Finally, the role of the hole-initiated processes with respect to electron-driven ionization is examined. Our results indicate the comparatively strong role of the former process, given that many traps within the wide bandgap $4 \mathrm{H}-\mathrm{SiC}$ would be empty.

\section{MODELING DETAILS}

The behavior of space-charge-limited current in high resistivity semiconductors containing discrete trapping levels was first discussed several decades ago. ${ }^{12,13}$ Since the early analytical treatments were inadequate, numerical approaches become necessary for a more complete and accurate analysis. ${ }^{14}$ Here, a numerical solution based on a one-dimensional (1D) rate equation model has been used, which incorporates self-consistent solutions of the Poisson equation at each time step. Three traps at energies of $0.8 \mathrm{eV}, 1.1 \mathrm{eV}$, and $1.2 \mathrm{eV}$ below the conduction band edge were used based on several 
reports, ${ }^{15-17}$ with total trap density assumed at $1 \times 10^{20} \mathrm{~m}^{-3}$. Within the bulk region, occupied trap densities before the start of the simulation were calculated from the following standard equation which provided the initial conditions:

$$
N_{T i}^{-}=\frac{N_{T i}}{\left(1+\frac{1}{2} e^{\frac{\left(E_{T i}-E_{f}\right)}{K T}}\right)},
$$

with $N_{T i}^{-}$and $N_{T i}$ denoting the occupied and total trap densities for the $i$ th trap level, respectively, $E_{T i}$ the trap level below the conduction band edge for the $i$ th trap, $k_{B}$ the Boltzmann constant, and $E_{f}$ the Fermi level. The rate equations for the analysis were taken as

$$
\begin{aligned}
& d n_{i} / d t= e_{n 0}(F) N_{T i}{ }^{-}-c_{n}(F) n\left(N_{T i}-N_{T i}{ }^{-}\right) \\
&+n N_{T i}{ }^{-} \exp \left[-K_{\text {electron }}\left(E_{c}-E_{T i}\right) /|F|\right] \beta_{n}\left|v_{n}\right|, \quad(2 \mathrm{a}) \\
& d p_{i} / d t= e_{p 0}(F)\left(N_{T i}-N_{T i}{ }^{-}\right)-c_{p}(F) p N_{T i}{ }^{-} \\
&+p\left(N_{T i}-N_{T i}{ }^{-}\right) \exp \left[-K_{\text {hole }}\left(E_{T i}-E_{v}\right) /|F|\right] \beta_{p}\left|v_{p}\right| \\
& d n(t) / d t=\sum_{i=1}^{3}\left(\frac{d n_{i}}{d t}\right) \\
& d p(t) / d t=\sum_{i=1}^{3}\left(\frac{d p_{i}}{d t}\right) \\
& d N_{T i}{ }^{-} / d t=-\left(\frac{d n_{i}}{d t}-\frac{d p_{i}}{d t}\right) .
\end{aligned}
$$

In the above, $F$ is the local electric field, $e_{n 0}$ and $e_{p 0}$ are the emission rates for electrons and holes, respectively, $d n_{i} / d t$ and $d p_{i} / d t$ symbolically represent the rate of mobile electron and hole density changes associated with the $i$ th level, $N_{T i}$ and $N_{T i}{ }^{-}$are the total and occupied trap densities for the $i$ th level, $v_{n, p}$ are the field-dependent drift velocities, $K_{\text {electron }}$ and $K_{\text {hole }}$ are constants for ionization from occupied traps due to electrons and holes, while $\beta_{n, p}$ are equivalent crosssections for the electron and hole rates. The last factor in Eqs. (2a) and (2b) represents the rate of electron- and holeassisted trap-to-band impact ionization.

Several authors have suggested an exponential dependence of the impact ionization coefficient on the local electric field $F$. This originates from the classic papers by Chynoweth, ${ }^{18}$ Shockley, ${ }^{19}$ Wolff, ${ }^{20}$ and later Baraff, ${ }^{21}$ which rest on deriving the probability for a carrier to gain energy from the electrical field exceeding the threshold energy. For a local model to be valid, two conditions must be fulfilled: (i) Every individual carrier has to sample a large energy space within the distribution, over a distance that is small compared to the mean distance between ionizing encounters. This is easy to satisfy at low to moderate electric fields, but could fail as ionization rates increase nonlinearly with field. (ii) The mean free paths have to be small compared to the spatial lengths over which electrical field changes take place. $^{22}$ If one of the above conditions is not satisfied, the "history" of the particle should be taken into account for calculating the ionization probability at any location. This is especially important over regions of rapidly changing electric fields and/or in small devices. In such situations, the energy gained by mobile carriers over a narrow spatial distance would likely be insufficient to cross the ionization threshold, resulting in the classical "dark space." 23 In this contribution, the non-local nature of the processes has been taken into account based on the "lucky-drift theory.",24,25 The central idea behind the "lucky-drift theory" is that a drifting electron could be "lucky" to reach an energy above the ionization threshold, as it undergoes both elastic and inelastic collisions. The field dependence for the electronand hole-ionization rates, as given in the last terms of Eqs. (2a) and (2b), was reported in the literature. ${ }^{24,25}$ In theory, other ways of treating the non-local element of ionization, in terms of the local carrier temperature, ${ }^{26-28}$ or Monte Carlo based distribution functions ${ }^{29,30}$ could be used. However, these techniques are numerically intensive and so were not implemented here for simplicity. Besides, expressions from the lucky-drift theory have been compared with more rigorous Monte Carlo simulations and found to yield good agreement. $^{31}$

The capture and emission rates were taken to be fielddependent as discussed in detail elsewhere. ${ }^{32}$ Very briefly, for an electric field $F$, the capture (or emission) rate coefficient $c_{n}$ (or $e_{n}$ ) can symbolically be written as $c_{n}(F)\left[=c_{n}\right.$ $\left.R_{c n}(F)\right]$ or $e_{n}(F)\left[=e_{n} R_{e n}(F)\right]$, where $R_{c n}(F)$ and $R_{e n}(F)$ represent field-dependent factors as given in Table I. The fielddependence for the capture coefficient arises since an applied field increases the average kinetic energy of electrons, making it easier for them to surmount a repulsive potential barrier. Alternatively, on the basis of the Lucovsky model, ${ }^{33}$ increased quantum mechanical tunneling of electrons through the potential barrier would increase the capture coefficients. Such electric field dependence of electron trapping was supported by the work of Sacks and Milnes, ${ }^{34}$ and the behavior of EL2 traps in GaAs. ${ }^{35}$ However, in the high electric field regime, carrier heating would cause the capture rate to decrease, since a long sequence of phonon emissions would then be required for the eventual and successful trapping of mobile carriers into the potential wells. ${ }^{36-38}$

For the present calculations, all concentrations (for both mobile carriers and traps) were taken to be spatially dependent, within a $0.454 \mathrm{~mm} 4 \mathrm{H}-\mathrm{SiC}$ device. A one-dimensional (1D) grid spacing $(\Delta \mathrm{x})$ of $14.64 \mu \mathrm{m}$ was used. This choice

TABLE I. Parameters used in the simulations.

\begin{tabular}{lc}
\hline \hline Fitting parameters & Value \\
\hline Hole injection ratio, $R_{i n j}$ & 0.05 \\
Emission co-efficient for electrons & $e_{v 0}=1.167 \times 10^{-16}$ \\
and holes, $e_{v}(F)=e_{v 0 *} R_{e}(F)$, & $R_{e}(F)=e^{F\left(\frac{\mathrm{v}}{\mathrm{m}}\right) 10^{-8} \mathrm{~m}^{3} / \mathrm{s}}$ \\
$v$ for electron or hole & \\
Capture co-efficient for electrons & $c_{v 0}=1.167 \times 10^{-16}$ \\
and holes, $c_{v}(F)=c_{v 0 *} R_{c}(F)$, & $R_{c}(F)=e^{F\left(\frac{\mathrm{v}}{\mathrm{m}}\right) 10^{-11} \mathrm{~m}^{3} / \mathrm{s}}$ \\
$v$ for electron or hole & \\
Electron ionization factor, $K_{\text {electron }}$ & $0.65 \times 10^{27}(\mathrm{C} \mathrm{m})^{-1}$ \\
Hole ionization factor, $K_{\text {holes }}$ & $0.62 \times 10^{26}(\mathrm{C} \mathrm{m})^{-1}$ \\
Equivalent cross section for electrons, $\beta_{n}$ & $8.07 \times 10^{-5} \mathrm{~m}^{2}$ \\
Equivalent cross section for holes, $\beta_{p}$ & $4.81 \times 10^{-17} \mathrm{~m}^{2}$ \\
\hline \hline
\end{tabular}


for the grid spacing satisfies the Courant-Friedrichs-Lewy (CFL) stability criteria. ${ }^{39}$ Two electrodes, the cathode (set at zero potential) and anode (subjected to the applied voltage), were taken to be on the left and right sides of the sample, respectively. For the $4 \mathrm{H}-\mathrm{SiC}$ material, the field-dependent details for electron ${ }^{40}$ and hole ${ }^{41}$ drift velocities $\left[v_{n}(F)\right.$ and $v_{p}(F)$, respectively] were taken from the literature. The analytical expression for electron velocity versus electric field (from Ref. 40) is given as

$$
\begin{aligned}
v_{n}(F)= & {\left[\mu_{n 1}|F|+\mu_{n 0}|F|\left(|F| / F_{0}\right)^{\theta}\right.} \\
& \left.+v_{\text {sat }}\left(|F| / F_{1}\right)^{\eta}\right] /\left[1+\left(|F| / F_{0}\right)^{\theta}+\left(|F| / F_{1}\right)^{\eta}\right],
\end{aligned}
$$

where $\quad F_{1}=2.8 \times 10^{7} \mathrm{~V} / \mathrm{m}, \quad F_{0}=3.05 \times 10^{6} \mathrm{~V} / \mathrm{m}, \quad \mu_{n 0}=4$ $\times 10^{-3} \mathrm{~m}^{2} / \mathrm{V} \mathrm{s}, \mu_{n 1}=0.68 \times 10^{-3} \mathrm{~m}^{2} / \mathrm{V} \mathrm{s}, \eta=3, v_{\text {sat }}=2.413$ $\times 10^{7} \mathrm{~m} / \mathrm{s}$, and $\theta=-1.95$. The hole drift velocity (in units of $\mathrm{m} / \mathrm{s}$ ), on the other hand, was approximated through a curve fit to a recent report for $\mathrm{SiC}^{41}$ and expressed in terms of the electric field $F$ (in units of $\mathrm{V} / \mathrm{m}$ ) as

$$
\begin{aligned}
v_{p}(F)= & \left(1.2 \times 10^{3} \times Z^{4}-4.1 \times 10^{3} \times Z^{3}+5.2\right. \\
& \left.\times 10^{3} \times Z^{2}-1.6 \times 10^{4} \times Z-4.7 \times 10^{4}\right),
\end{aligned}
$$

where $Z=\frac{\left(F-8.2 \times 10^{6}\right)}{5.9 \times 10^{6}}$.

The possibility of hole injection from the anode following thermalization of the highly energetic electrons collected at the contact was also included. Figure 1 shows a schematic for the process of hole injection from the anode. Basically, electrons exiting the $\mathrm{SiC}$ sample and incident on the anode contact have a distribution of energies and thermalize through momentum and energy exchange within the metal. Though the low-energy electrons would not produce electron-hole pairs or plasmons within the metal, the more energetic electrons could contribute to hole generation as a result of the energy exchanges. The holes so produced would have a distribution of energies. Of these, some would successfully tunnel back into the $\mathrm{SiC}$ valence band and contribute to device current. Hence, quite simply, the injected hole flux can be expected to be some fraction of the incoming electron flow, i.e., $J_{p}=J_{n} R_{i n j}(F)$, where $J_{p}$ and $J_{n}$ denote the

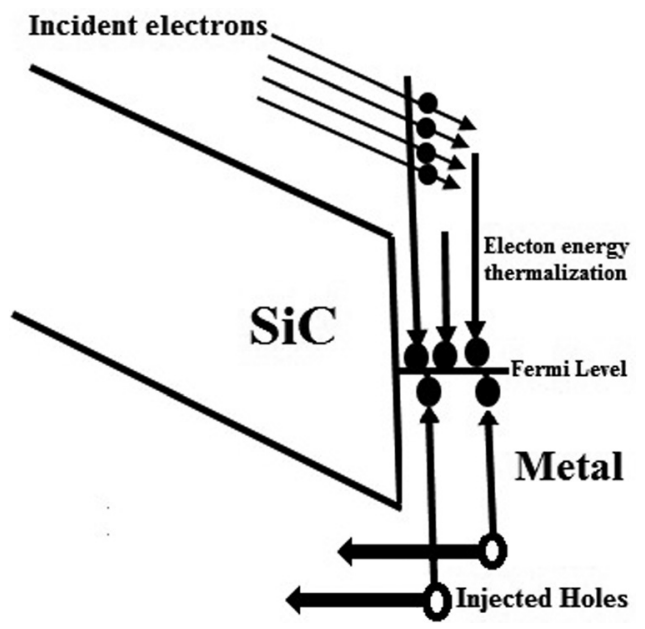

FIG. 1. Schematic of hole injection at the anode following thermalization of the incident distribution of high energy electrons collected at the contact. hole and electron current densities, and $R_{i n j}(F)$ is a fielddependent factor that folds in the probability of hole creation and their subsequent injection into the valence band. An accurate determination for $\mathrm{R}_{\mathrm{inj}}(\mathrm{F})$ requires the energy distribution for the electron swarm, as possible from Monte Carlo simulations. ${ }^{42}$ However, for simplicity, $R_{i n j}(F)$ was taken to be a field-dependent adjustable parameter.

\section{SIMULATION RESULTS AND DISCUSSION}

The above one-dimensional, time-dependent model was used to evaluate the response of a $0.454 \mathrm{~mm}$ thick $4 \mathrm{H}-\mathrm{SiC}$ sample containing traps to a $6 \mathrm{kV}$ voltage step in the absence of any external photoexcitation. Three trap levels at 0.8 , 1.1 , and $1.2 \mathrm{eV}$ below the conduction band edge were simultaneously assumed. Relevant parameters used have been summarized in Table I. Results for trap-to-band impact ionization are discussed first. Figure 2 for the field-dependent impact ionization coefficients shows the stronger role of the hole-initiated process as compared to electron-initiated ionization. This implies that even without any photoexcitation to create electron-hole pairs, holes could be generated in the wide bandgap material containing defects at moderately high fields. The dominance of the hole-initiated ionization can be understood from the last terms of Eqs. (2a) and (2b). The occupancy of traps is typically low in the wide bandgap material. With most levels unoccupied, the process of placing electrons in the conduction band from the traps is weak. Hot or energetic holes moving in the valence band can lead to Auger processes, wherein they release their energy enabling electron transitions from the valence band into unoccupied traps to leave behind holes. In Figure 2, with increasing electric field, the band-to-trap ionization rates are seen to increase monotonically, while the disparity between the hole- and electron-initiated processes decreases. The field-dependent increases result from the exponential factors in Eq. (2). In addition, increases in the current (hence particle densities) work to boost the ionization rates. Higher carrier densities within the $\mathrm{SiC}$ with increasing current help enhance the trap occupancy as the sample gets progressively flooded with mobile charge at higher fields.

The role of hole injection is examined in the next set of results, which were again obtained without any photoexcitation. In the absence of Monte Carlo simulations for

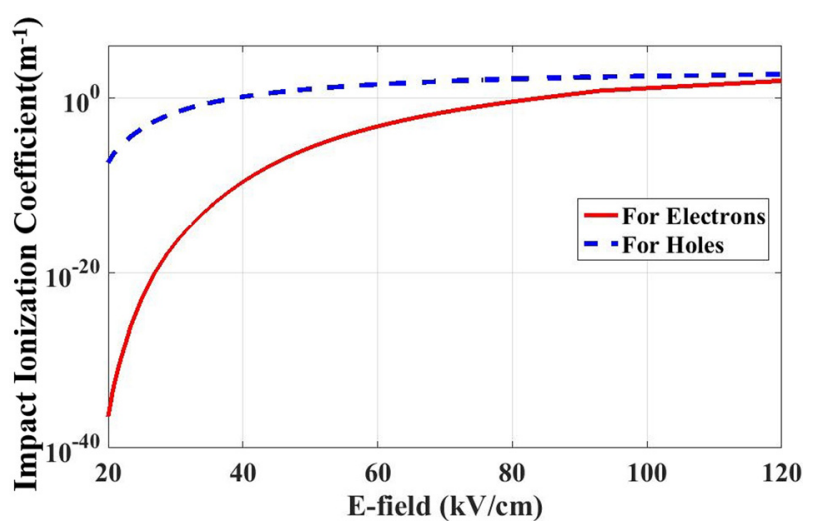

FIG. 2. Trap-to-band impact ionization coefficients as a function of electric field. The hole process is predicted to dominate over the electron route. 
consistency, the hole injection was taken to be a parameter, based on the $R_{i n j}$ term of Sec. II. Snapshots of the simulation results obtained for the electron density within the $\mathrm{SiC}, 30 \mathrm{~ns}$ after applying the voltage step at the anode, with and without the inclusion of anode hole injection, are shown in Figs. 3(a) and 3(b), respectively. The parameter $R_{i n j}$ was assigned a low value of 0.05 as it would lead to reasonable comparisons with experimental data, as discussed later. The impact ionization processes were operative for both cases. Results reveal that without hole injection, the profile is relatively flat and much lower as compared to the case shown in Fig. 3(b). Hole injection alters the overall situation in the following two ways. Hole-initiated impact ionization is enhanced. This in turn keeps supplying electrons to the trap levels (by raising the trap occupancy densities $\mathrm{N}_{\mathrm{Ti}}{ }^{-}$). This sequentially fuels electron occupancy in the conduction band via the electron-initiated trap-to-band impact ionization and trap emission processes. The rates increase with electron density, making it more dominant towards the anode on the right. Thus, the electron density profile of Fig. 3(b) is seen to have higher concentrations than that of Fig. 3(a). The monotonic increase in electron density towards the anode arises from the movement of ever-increasing numbers of electrons drifting to the anode. In any event, the results highlight the important role of hole injection in influencing the sample conductivity in semi-insulating $\mathrm{SiC}$. With photoexcitation, this process can be expected to play an even more significant role than probed here.
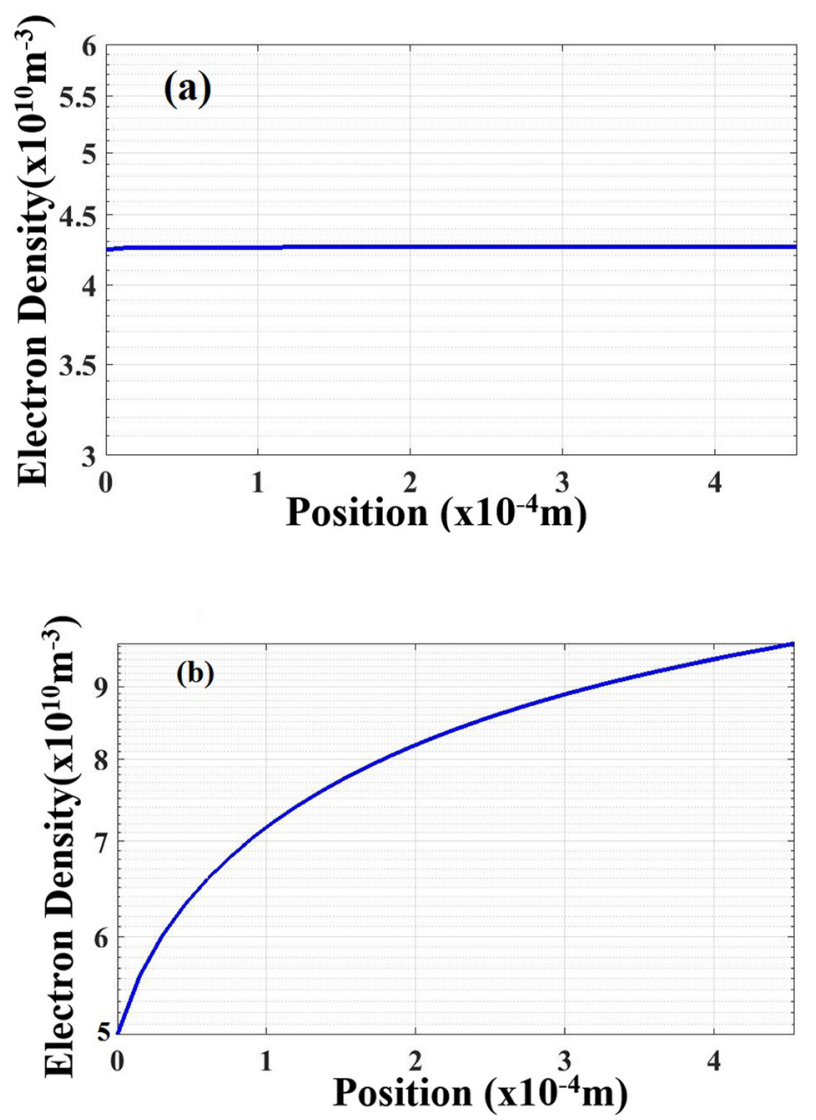

FIG. 3. A snapshot 30-ns after applying a voltage step showing the electron density profile with inclusion of impact ionization. (a) Without hole injection from the anode and (b) with inclusion of hole injection from the anode.
The electron profile shown in Fig. 3(b) shapes the spatial trap occupancy distribution. This is evident in Fig. 4 which is a snapshot obtained $30 \mathrm{~ns}$ after applying the device voltage. The curve shows the occupancy of the $0.8 \mathrm{eV}$ trap (though all three trap levels were included in the simulations), as a function of position for the case with hole injection. With the electron density increasing towards the right (as in Fig. 3(b)), the electron initiated impact ionization is enhanced, leading to a progressively stronger depletion of electrons from the trap states towards the anode end on the right.

Next, the time-dependent device current density response to a $6 \mathrm{kV}$ voltage step was probed. The simulation results obtained are shown in Fig. 5. The three curves correspond to calculations with: (a) both impact ionization and hole injection included, (b) with impact ionization but no hole injection, and (c) no impact ionization but with hole injection. The negative current values in Fig. 5 are simply due to the chosen polarity. The cathode was at the left side and the anode on the right, yielding a current flow from right-to-left. The curves with impact ionization exhibited a sharper rise, as compared to the simulation result without impact ionization, to a magnitude of around $1 \mathrm{~A} / \mathrm{m}^{2}$. In Fig. 5 , the current is the lowest in the absence of impact ionization, thus underscoring the importance of the trap-to-band impact ionization process. The general trend seen in the figure is for a very rapid initial increase in current as the voltage is turned on, followed by a drop as mobile charge is sweptout of the device and the displacement current falls. With impact ionization included, the currents are higher and the largest value occurs for the case when anode hole injection is also included. The hole-initiated trap-to-band process is triggered by the injected holes. The current fluctuations predicted in Fig. 5 with inclusion of hole injection from the anode contact deserve some explanation. A simple qualitative scenario is as follows. Hole injection (with hole-initiated impact ionization) leads to a free-carrier density increase, but this enhancement then reduces the electric field due to the higher conductivity. This field correction weakens impact ionization and thus the source term for current generation. Carrier loss through sweep-out continues however and works to lower the local free-carrier density. Over time, the carrier loss leads to increased local electric fields from their

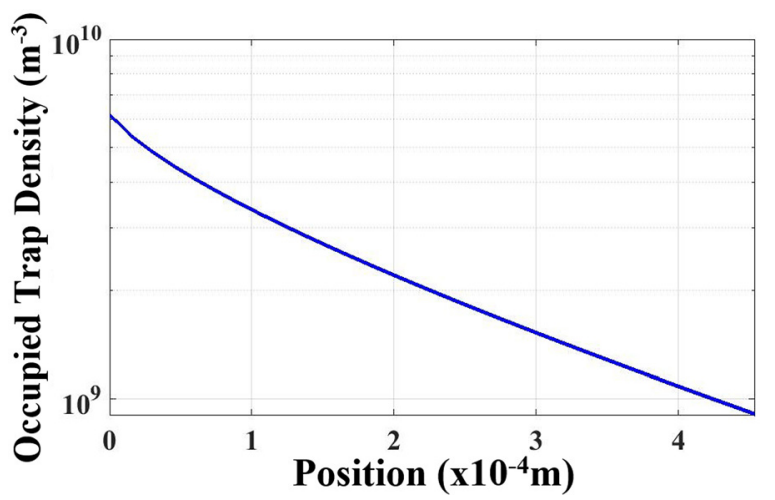

FIG. 4. A snapshot of the occupied trap profile for the $0.8 \mathrm{eV}$ level below the conduction band after 30 -ns from the start of the voltage. 


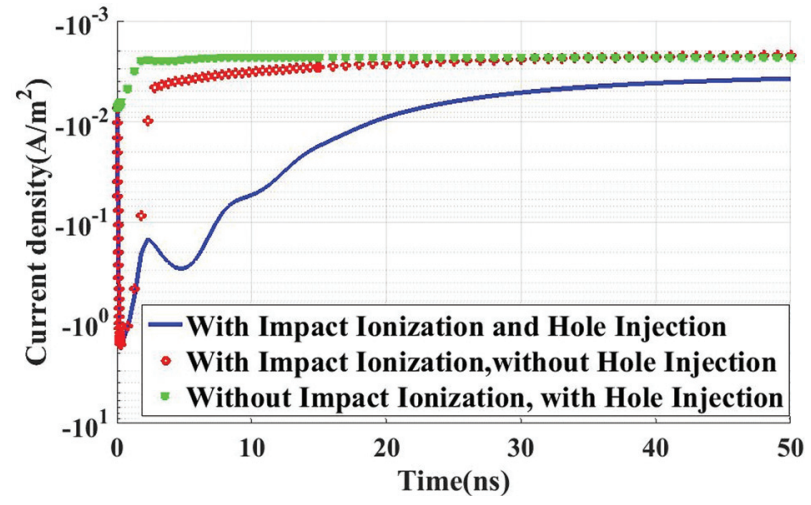

FIG. 5. Simulation result for the time-dependent current response to a $6 \mathrm{kV}$ voltage step applied to the semi-insulating $4 \mathrm{H}-\mathrm{SiC}$ sample.

depressed values and strengthens impact ionization since this process is non-linearly dependent on the field magnitude. In addition, the presence of hole-injection contributes to filling the unoccupied traps as a result of hole initiated impact ionization. This also helps increase the electron initiated impact ionization, collectively leading to current increases. However with continued sweep-out over longer times, the charge densities continue to decrease and so any fluctuation is predicted to cease within about $13 \mathrm{~ns}$ as the current progresses towards a steady-state.

The above reasoning in the context of time-dependent variations is further validated from an assessment of changes in the electron ionization co-efficient for the three different trap levels over time. Figure 6 shows oscillation in the ionization rates at the mid-point of the device as a result of dynamical changes in both the trap occupancies and local carrier densities that initiate ionization. The dielectric relaxation, trapping-detrapping dynamics, and ionization rates collectively govern the temporal evolution.

Finally, the time-dependent simulations were extended to long times until steady-state at different applied voltages having magnitudes ranging from $454 \mathrm{~V}$ to $7200 \mathrm{~V}$. This yielded steady-state characteristics of the current density versus spatially averaged device field $(J-E)$ for the chosen $4 \mathrm{H}-\mathrm{SiC}$ model. The results are shown in Fig. 7, along with experimental data obtained from our laboratory. For the

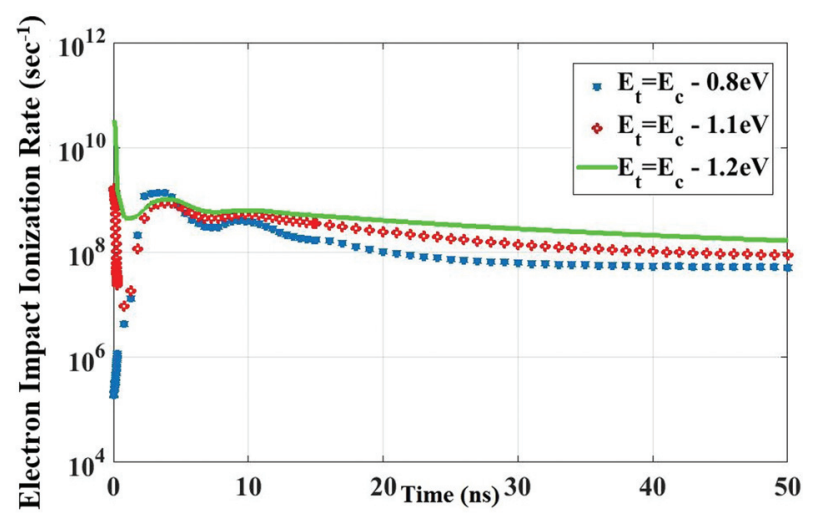

FIG. 6. Calculated changes in the electron initiated impact ionization coefficient for three different trap levels $(0.8 \mathrm{eV}, 1.1 \mathrm{eV}$, and $1.2 \mathrm{eV}$ below the conduction band edge) over time. simulations, hole injection was always implemented, though in one case trap-to-band ionization processes were turned off. The curves show that an absence of impact ionization is inadequate and does not lead to a good match with the experimental results. Inclusion of impact ionization and hole injection certainly yields much better results and compares well with the data. Results with impact ionization but without hole injection were similar to those without impact ionization but with hole injection and are not been shown.

\section{CONCLUSIONS}

Application of high voltages is necessary and germane to pulsed power systems. Hence, semiconductor devices that are used for electronic control and switching are routinely subjected to high electric fields. Their operation in this regime needs to be understood for performance optimization and to avoid deleterious effects. Here, we have reported our simulation studies of high field conduction in $4 \mathrm{H}-\mathrm{SiC}$ containing traps for switching applications. For simplicity, the response has been analyzed in the absence of external photoexcitation, though the model can easily be extended to include carrier generation. The motivation for the present study arises from the need to probe the influence of trap-toband impact ionization since semi-insulating $\mathrm{SiC}$ typically contains traps. Most previous studies have focused on bandto-band impact ionization, which requires much higher threshold fields and may not be as important. Besides ionization, the role of hole injection from the anode was also explicitly included.

Our simulation results show that hole-initiated processes can be important given that the trap levels would be unoccupied. Our results yielded reasonable agreement with measured current-voltage data, but only when impact ionization was taken into account. The defects in such devices would limit leakage currents and enhance hold-off voltages, while affording the possibility for high device conductance in the on-state from trap-initiated impact ionization processes. The agreement between the model and experiments obtained was quite reasonable and extended over three orders of magnitude. Arguably, a slightly different set of material parameters could also have yielded similar results. So while we do not

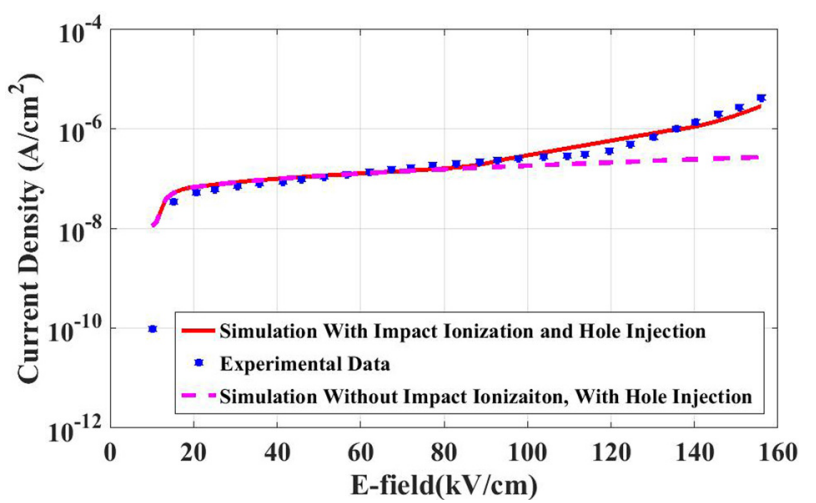

FIG. 7. Steady state J-E curves obtained from time-dependent simulations under two different conditions carried out over long times in response to voltage steps of different amplitudes. The experimental data points obtained by our group are also shown for comparison. 
claim uniqueness, the basic physical mechanisms and trends shown here would be expected to hold. A possible SiC-based PCSS for the present pulsed power applications would probably incorporate a deep trap at around $1.2 \mathrm{eV}$ below the conduction band to minimize the dark currents, while contributing to trap-to-band impact ionization during the turn-on phase of the switch. Neutron irradiation would likely be useful from the standpoint of spatial uniformity of the traps. Furthermore, a nickel anode contact would serve to provide for hole injection, given the relatively higher electron barrier height for nickel. Finally, our time-dependent simulations have predicted undulations in the device conduction current and suggest that the behavior likely arises from a dynamic interplay between impact ionization, electric field values, and occupancies of the trap levels controlled by trapping-detrapping.

For completeness, it may be noted that Monte Carlo simulation could be performed as a next step to obtain precise hole injection currents and their energy-dependent probabilities from knowledge of the electron distribution arriving at the anode. However, an accurate analysis would require an in-depth evaluation of the various trap levels, especially in the vicinity of the anode contact in order to fold in the tunneling of holes into the valence band. Similarly, adding heating and temperature-dependent variations in the transport parameters to the model would be an important step towards a more complete analysis. Finally, extending the analysis to two dimensions with inclusion of temperature dependent parameters and local trap density fluctuations could help probe stability issues.

\section{ACKNOWLEDGMENTS}

The work was supported in part from by the Office of Naval Research under Grant No. N00014-15-1-2650 and by AFOSR Grant No. FA95501010106, “Collaborative Research on Novel High Power Sources and Physics of Ionospheric Modification."

${ }^{1}$ D. H. Auston, Appl. Phys. Lett. 26, 101 (1975).

${ }^{2}$ G. J. Caporaso, Y. J. Chen, and S. E. Sampayan, Rev. Accel. Sci. Technol. 2, 253 (2009).

${ }^{3}$ J. W. B. Bragg, W. W. Sullivan III, D. Mauch, A. A. Neuber, and J. C. Dickens, Rev. Sci. Instrum. 84, 054703 (2013).

${ }^{4}$ M. Bhatnagar and B. J. Baliga, IEEE Trans. Electron Devices 40, 645 (1993).

${ }^{5}$ H. Morkoç, S. Strite, G. B. Gao, M. E. Lin, B. Sverdlov, and M. Burns, J. Appl. Phys. 76, 1363 (1994).
${ }^{6}$ Properties of Advanced Semiconductor Materials, edited by M. E. Levinshtein, S. L. Rumyantsev, and M. S. Shur (Wiley, New York, 2001). ${ }^{7}$ B. J. Baliga, Advanced Power MOSFET Concepts (Springer, New York, 2010).

${ }^{8}$ A. Ogunniyi, H. O’Brien, A. Lelis, C. Scozzie, W. Shaheen, A. Agarwal, J. Zhang, R. Callanan, and V. Temple, Solid State Electronics 54, 1232 (2010).

${ }^{9}$ K. S. Kelkar, N. E. Islam, C. M. Fessler, and W. C. Nunnally, J. Appl. Phys. 100, 124905 (2006).

${ }^{10}$ S. Dogan, A. Teke, D. Huang, H. Morkoc, C. B. Roberts, J. Parish, B. Ganguly, M. Smith, R. E. Myers, and S. E. Saddow, Appl. Phys. Lett. 82, 3107 (2003).

${ }^{11}$ F. Zhao, M. M. Islam, P. Muzykov, A. Bolotnikov, and T. S. Sudarshan, IEEE Electron Device Lett. 30, 1182 (2009).

${ }^{12}$ A. Rose, Phys. Rev. 97, 1538 (1955).

${ }^{13}$ M. A. Lampert, A. Rose, and R. W. Smith, J. Phys. Chem. Solids 8, 464 (1959).

${ }^{14}$ G. H. Dohler and H. Heyszenau, Phys. Rev. B 12, 641 (1975).

${ }^{15}$ W. C. Mitchela, W. D. Mitchell, H. E. Smith, G. Landis, S. R. Smith, and E. R. Glaser, J. Appl. Phys. 101, 053716 (2007).

${ }^{16}$ N. Sghaier, J.-M. Bluet, A. Souifi, G. Guillot, E. Morvan, and C. Brylinski, IEEE Trans. Electron Devices 50, 297 (2003).

${ }^{17}$ M. V. S. Chandrashekhar, I. Chowdhury, P. Kaminski, R. Kozlowski, P. B. Klein, and T. Sudarshan, Appl. Phys. Express 5, 025502 (2012).

${ }^{18}$ A. G. Chynoweth, Phys. Rev. 109, 1537 (1958).

${ }^{19}$ W. Shockley, Solid-State Electron. 2, 35 (1961).

${ }^{20}$ P. A. Wolff, Phys. Rev. 95, 1415 (1954).

${ }^{21}$ G. A. Baraff, Phys. Rev. 133, A26 (1964).

${ }^{22}$ E. E. Kunhardt, J. Wu, and B. Penetrante, Phys. Rev. A 37, 1654 (1988).

${ }^{23}$ Y. Okuto and C. R. Crowell, Phys. Rev. B 10, 4284 (1974).

${ }^{24}$ B. K. Ridley, J. Phys. C: Solid State Phys. 16, 3373 (1983).

${ }^{25}$ S. McKenzie and M. G. Burt, J. Phys. C: Solid State Phys. 19, 1959 (1986).

${ }^{26} \mathrm{~K}$. Katayama and T. Toyabe, "A new hot carrier simulation method based on full 3d hydrodynamic equations," in Technical Digest International Electron Devices Meeting (IEDM), 1989, pp. 135-138.

${ }^{27}$ Y. Apanovich, E. Lyumkis, B. Polsky, A. Shur, and P. Blakey, IEEE Trans. Comput.-Aided Des. Integr. Circuits Syst. 13, 702 (1994).

${ }^{28}$ M. Ershov and V. Ryzhii, Semicond. Sci. Technol. 10, 138 (1995).

${ }^{29}$ Y. Kamakura, H. Mizuno, M. Yamaji, M. Morifuji, K. Taniguchi, C. Hamaguchi, T. Kunikiyo, and M. Takenaka, J. Appl. Phys. 75, 3500 (1994).

${ }^{30}$ R. L. Kamocsai and W. Porod, J. Appl. Phys. 69, 2264 (1991).

${ }^{31}$ S. McKenzie and M. G. Burt, Semicond. Sci. Technol. 2, 275 (1987).

${ }^{32}$ R. Tiskumara, R. P. Joshi, D. Mauch, J. C. Dickens, and A. A. Neuber, J. Appl. Phys. 118, 095701 (2015).

${ }^{33}$ G. Lucovsky, Solid State Commun. 3, 299 (1965).

${ }^{34}$ H. K. Sacks and A. G. Milnes, Int. J. Electron. 28, 565 (1970).

${ }^{35}$ F. Piazza, P. C. M. Christianen, and J. C. Maan, Appl. Phys. Lett. 69, 1909 (1996).

${ }^{36}$ R. P. Joshi, J. Appl. Phys. 74, 1810 (1993).

${ }^{37}$ M. Lax, Phys. Rev. 119, 1502 (1960).

${ }^{38}$ L. Reggiani, L. Varani, V. V. Mitin, and C. M. Van Vliet, Phys. Rev. Lett. 63, 1094 (1989).

${ }^{39}$ R. Courant, K. Friedrichs, and H. Lewy, IBM J. Res. Dev. 11, 215 (1967).

${ }^{40}$ H. Lv, Y. Zhang, Y. Zhang, and L. A. Yang, IEEE Trans. Electron Devices 51, 1065 (2004).

${ }^{41}$ A. Akturk, N. Goldsman, S. Potbhare, and A. Lelis, J. Appl. Phys. 105, 033703 (2009).

${ }^{42}$ For example, R. P. Joshi and D. K. Ferry, Solid State Electron. 38, 1911 (1995). 